\title{
1 An interplay between plasticity and parental phenotype determines impacts of ocean acidification on a reef fish
}

4 Celia Schunter, Megan J. Welch, Göran E. Nilsson, Jodie L. Rummer, Philip L. Munday* and Timothy Ravasi*

\section{$7 \quad$ Introductory paragraph}

8 The impacts of ocean acidification will depend on the ability of marine organisms to

9 tolerate, acclimate, and eventually adapt to changes in ocean chemistry. Here, we use a

10 unique transgenerational experiment to determine the molecular response of a coral reef

11 fish to short-term, developmental, and transgenerational exposure to elevated $\mathrm{CO}_{2}$ and to

12 test how these responses are influenced by variations in tolerance to elevated $\mathrm{CO}_{2}$

13 exhibited by the parents. Within-generational responses in gene expression to end of

14 century predicted $\mathrm{CO}_{2}$ levels indicate that a self-amplifying cycle in GABAergic

15 neurotransmission is triggered, explaining previously reported neurological and

16 behavioural impairments. Furthermore, epigenetic regulator genes exhibited a within-

17 generation specific response, but with some divergence due to parental phenotype.

18 Importantly, we find that altered gene expression for the majority of within-generation

19 responses returns to baseline levels following parental exposure to elevated $\mathrm{CO}_{2}$

20 conditions. Our result show that both parental variation in tolerance and cross-generation

21 exposure to elevated $\mathrm{CO}_{2}$ are crucial factors in determining the response of reef fishes to

22 changing ocean chemistry. 
24 Keywords: Developmental plasticity, Parental effects, Epigenetic regulation, Ocean

25 acidification, Transcriptomics, Adaptation.

\section{Introduction}

28 Increased uptake of anthropogenic $\mathrm{CO}_{2}$ by the oceans and the seawater acidification it

29 causes will have detrimental effects on many marine organisms ${ }^{1}$. Laboratory experiments

30 have already provided evidence of a diverse range of responses and effects of ocean

31 acidification conditions ${ }^{2-4}$, including altered growth rates, survival, and reproduction ${ }^{5,6}$.

32 Fish and other marine organisms can also exhibit behavioural changes that could affect

33 survivorship ${ }^{7,8}$, including vital responses to chemical alarm and predator cues ${ }^{9-14}$. The

34 underlying cause of these behavioural impairments is thought to be changed

35 concentrations of acid-base relevant ions to prevent acidosis under elevated $\mathrm{CO}_{2}$, which

36 in turn affects the function of gamma-aminobutyric acid (GABA) neurotransmitter

37 receptors in the brain ${ }^{14-16}$.

39 To date, most observations regarding impacts of ocean acidification come from short-

40 term experiments that do not account for population heterogeneity and individual

41 variation in tolerance potentially important to adaptive processes ${ }^{17,18}$. Acutely exposing

42 animals to elevated $\mathrm{CO}_{2}$ for days to weeks cannot predict the potential for long-term

43 acclimation and adaptation ${ }^{18}$. In particular, conditions experienced early in life can affect

44 responses to those conditions later in life (i.e., developmental plasticity), which can be

45 mediated by epigenetic modifications ${ }^{19}$. The environment experienced by the parents can

46 also influence how offspring respond ${ }^{20-22}$. In fact, recent transgenerational studies 
47 demonstrate recovery of metabolic and growth rates in juvenile fish when both parents

48 and offspring are exposed to elevated $\mathrm{CO}_{2}{ }^{23,24}$. Finally, individual variation in $\mathrm{CO}_{2}$

49 tolerance could be heritable, and therefore, variation in parental tolerance to elevated $\mathrm{CO}_{2}$

50 could influence the tolerance of their offspring ${ }^{25}$. Longer-term developmental studies and

51 multigenerational experiments that incorporate individual variation in tolerance are

52 needed to better understand and predict the effects of elevated $\mathrm{CO}_{2}$ on populations and

53 their capacity to adapt ${ }^{17,26}$.

54

55 A recent brain transcriptome study on juvenile spiny damselfish (Acanthochromis

56 polyacanthus) exposed to elevated $\mathrm{CO}_{2}$ revealed phenotypic differences between

57 offspring of parents with behavioural tolerance versus sensitivity to elevated $\mathrm{CO}_{2}{ }^{27}$. This

58 suggests that parental phenotype could influence the expression of developmental and

59 transgenerational plasticity to elevated $\mathrm{CO}_{2}$ in reef fishes. To further understand the

60 mechanisms that underpin this plasticity, we investigated the effects of acute, long-term

61 developmental, and transgenerational exposure to elevated $\mathrm{CO}_{2}$ on the molecular

62 response of juvenile spiny damselfish from behaviourally tolerant and sensitive parents.

63 We focus on the brain because altered function of $\mathrm{GABA}_{\mathrm{A}}$ neurotransmitter receptors are

64 thought to be responsible for many behavioural changes observed in fish exposed to

65 elevated $\mathrm{CO}_{2}{ }^{15,16}$. Adult spiny damselfish were exposed to a near-future $\mathrm{CO}_{2}$ level

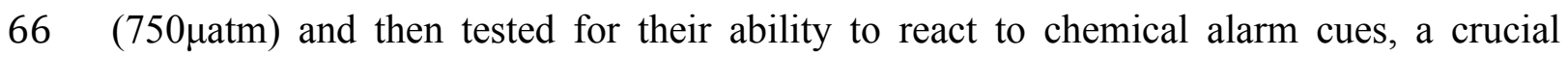

67 survival mechanism in fish ${ }^{11}$. Based on these results, adults were matched into

68 behaviourally 'tolerant' and 'sensitive' breeding pairs that were maintained under either

69 current-day or elevated $\mathrm{CO}_{2}$ (Methods \& Figure 1). Offspring of these pairs were reared 
70 under both control and elevated $\mathrm{CO}_{2}$ conditions for 5 months. Finally, some offspring

71 reared under control conditions from hatching were exposed to elevated $\mathrm{CO}_{2}$ for the last 4

72 days of the experiment. This produced four different treatments for the two parental

73 phenotypes: a) control $\mathrm{CO}_{2}$ parents - offspring reared in control conditions (control); b)

74 control $\mathrm{CO}_{2}$ parents - offspring reared in control conditions, but with a final 4-day

75 elevated $\mathrm{CO}_{2}$ treatment at the age of 5 months (acute $\mathrm{CO}_{2}$ treatment), c) control $\mathrm{CO}_{2}$

76 parents - offspring reared in elevated $\mathrm{CO}_{2}$ from hatching (developmental $\mathrm{CO}_{2}$ treatment);

77 d) elevated $\mathrm{CO}_{2}$ parents - offspring reared in elevated $\mathrm{CO}_{2}$ from hatching

78 (transgenerational $\mathrm{CO}_{2}$ treatment) (Figure 1). We measured the genome-wide gene

79 expression in the brains of 72 individuals across all treatments to tease apart the acute

80 response to elevated $\mathrm{CO}_{2}$ from the responses to longer-term development under elevated

$81 \mathrm{CO}_{2}$ and differences that occur due to parental exposure to elevated $\mathrm{CO}_{2}$. Comparing

82 these transcriptomes in offspring from two parental phenotypes allowed us to evaluate

83 how long-term and cross-generational exposure to elevated $\mathrm{CO}_{2}$ influences the response

84 of fish to future ocean acidification conditions and the influence of individual variation in

85 tolerance to elevated $\mathrm{CO}_{2}$ on these relationships.

86 


\section{$87 \quad$ Results}

\section{Influence of parental phenotype on the response to elevated $\mathrm{CO}_{2}$}

89 The offspring of behaviourally tolerant and sensitive parents exhibited significant

90 differences in the brain transcriptome. We identified 114 differentially expressed

91 transcripts (DETs) under acute $\mathrm{CO}_{2}$ exposure and 359 under developmental exposure

92 when comparing offspring from the two parental groups directly, revealing a clear

93 influence of the parental phenotype on the offspring's response to elevated $\mathrm{CO}_{2}($ Figure 2,

94 Supplementary Figure 1). The DETs expressed between offspring of the two parental

95 phenotypes upon acute exposure were functionally enriched in pathways controlling

96 haemoglobin and oxygen transport (Supplementary Data 1). No significantly enriched

97 function was found for DETs between parental phenotypes in the developmental 98 treatment.

100 Besides direct differential expression between offspring of the two parental phenotypes,

101 we also compared expression within each parental group (e.g. acute treatment versus

102 control) in order to identify transcripts with expression profiles that overlap or differ

103 between the two parental phenotypes. While there were similarities, there were also large

104 differences in gene expression patterns among treatments for the offspring of tolerant and

105 sensitive parents (Supplementary Data 2). Offspring of behaviourally tolerant parents

106 exhibited more changes in the transcriptome when acutely exposed to elevated $\mathrm{CO}_{2}$

107 (3,669 DETs) compared to the developmentally exposed fish (1,142 DETs) (Figure 2).

108 Interestingly, this pattern was reversed in the offspring of sensitive parents, where the

109 developmental treatment exhibited mores change in gene expression (2,590 DETs) 
110 compared with the acute treatment (2,010 DETs). The shared component between the

111 parental phenotypes for these treatments was as low as $27 \%$, and few pathways were

112 commonly enriched in the brains of fish from different parental phenotypes in the

113 developmental treatment (Figure 3). In the developmental treatment, only offspring of

114 tolerant fish showed differential expression of transcripts involved in gluconeogenesis.

115 Several other pathways were enriched only in the offspring of behaviourally sensitive

116 parents, including pathways involved in nervous system development and ion transport

117 (Supplementary Data 3). We therefore found large differences, yet some overlapping

118 transcriptional responses in the offspring of the two parental phenotypes. Nonetheless,

119 the acute and developmental $\mathrm{CO}_{2}$ treatments had larger overall effects on the

120 transcriptome than did the parental phenotype (Supplementary Figure 1).

122 Short-term and developmental responses to elevated $\mathrm{CO}_{2}$

123 Exposure of offspring to a near-future elevated $\mathrm{CO}_{2}$ level resulted in large differences in 124 gene expression compared with control offspring reared at the current-day $\mathrm{CO}_{2}$ level

125 (Figure 2). Offspring of behaviourally tolerant parents that were acutely exposed to

126 elevated $\mathrm{CO}_{2}$ for 4 days exhibited the greatest number of DETs $(3,669)$ compared to

127 control fish (14.5\% of the entire brain transcriptome). In this acute treatment, about half

128 of the DETs (51\% and $49 \%$ for offspring of tolerant and sensitive parents respectively)

129 were expressed at higher levels and resulted in more significant functional enrichments

130 than the transcripts upregulated in controls (Figure 3). Comparing DETs in the acute

131 treatment with those differentially expressed in longer-term treatments enabled us to

132 distinguish rapid, short-term from longer-term transcriptional responses to elevated $\mathrm{CO}_{2}$. 
133 For this comparison we considered the transcripts that were differentially expressed in

134 acutely-treated compared with control fish, but which were not differentially expressed in

135 developmental and transgenerationally treated fish compared to controls. Hence, these

136 DETs were unique to the acute 4-day elevated $\mathrm{CO}_{2}$ treatment. A total of 184 genes

137 showed a clear pattern of specific short-term response that was common for both parental

138 phenotypes (Supplementary Data 4). These acute-specific genes were significantly

139 enriched in ATPase-related processes (Figure 3 \& Supplementary Data 5).

141 Fish that were developmentally exposed to elevated $\mathrm{CO}_{2}$ differentially expressed 1,142

142 and 2,590 transcripts, of which $56 \%$ and $78 \%$ were upregulated in offspring of tolerant

143 and sensitive parents, respectively (Figure 2). The offspring of sensitive parents had a

144 large number of enriched biological pathways that showed upregulation in the

145 developmental treatment (Figure 3). A total of 698 transcripts were commonly

146 differentially expressed in offspring of both parental phenotypes. Only 27 of these

147 transcripts were uniquely differentially expressed in the developmental $\mathrm{CO}_{2}$ treatment,

148 regardless of parental phenotype, suggesting developmental treatment specificity

149 (Supplementary Data 6). These transcripts were at control expression levels in acute and

150 transgenerational treatments, but differentially expressed in the developmental treatment.

151 Of these transcripts, 23 showed downregulated expression in the developmental treatment

152 when compared to the controls.

153

154 Importantly, in both the acute and developmental treatments we found a common set of

155 highly upregulated transcripts involved in neurotransmitter secretion, nervous system

156 development, ionotropic glutamate receptor activity, and $\mathrm{GABA}_{\mathrm{A}}$ receptor activity 
157 (Figure 3). This upregulation was specific to within-generation treatments, including

158 acutely exposed fish and fish reared under elevated $\mathrm{CO}_{2}$ for 5 months from hatching.

159 Many of these DETs and associated enriched functions were also found in a weighted

160 correlation network gene cluster (Supplementary Data 7). Hence, both of these

161 independent methods revealed the importance of these genes and functions for fish

162 exposed to elevated $\mathrm{CO}_{2}$. A clear signature came from GABAergic neurotransmission,

163 with nearly all genes in this pathway overexpressed in the acutely and developmentally

164 treated fish when compared to controls (Figure 4). These included genes involved in

165 GABA production, GABA secretion from presynaptic neurons, all of the $\mathrm{GABA}_{\mathrm{A}}$

166 receptor subunits (Supplementary Data 8), and the potassium-chloride co-transporter 2

$167(k c c 2)$. Furthermore, we saw a reduction in the expression of GABA transporter 1 (gat 1 ).

169 Another within-generation specific response involved epigenetic regulation of gene 170 expression. Here we saw common, but also divergent, responses between the parental

171 phenotypes. In the developmental treatment, there were significant differences in the 172 expression of genes involved in methylation between the offspring from different 173 parental groups. Specifically, eight DETs from the direct comparison between the 174 parental groups in the developmental treatment are involved in the control of the DNA,

175 protein, and histone methylation states (ppmel, apexl, prmt6, setd2, $k m t 2 a$, mecp $2, k m t 2 c$

$176 \& \mathrm{mrm} 1$ ) (Supplementary Data 9). Differences in epigenetic related transcription patterns

177 could also be seen across different $\mathrm{CO}_{2}$ treatments, as methylation related pathways were

178 significantly enriched in genes that were downregulated in the offspring of tolerant 179 parents, but only when offspring were acutely exposed to elevated $\mathrm{CO}_{2}$. 
181 Transcripts encoding histones also showed treatment-specific expression when

182 considering the parental phenotypes. In the acute treatment, two isoforms of histone 1

$183(h 1 b, h 10)$ were highly expressed in offspring of behaviourally sensitive parents (Figure

184 5a), but not in the offspring of tolerant parents. However, the expression for other histone

185 variants seemed treatment-specific in fish acutely and developmentally exposed to

186 elevated $\mathrm{CO}_{2}$, regardless of the parental phenotype (Figure 5a). In general, the expression

187 levels of histones were lower in fish from the developmental treatment for offspring of

188 both parental phenotypes. It is, however, important to note that histone modifiers (e.g.,

189 histone-lysine methyltransferases; setd2, $k m t 2 a, k m t 2 c$ ) were upregulated in the

190 developmental treatment for offspring of tolerant parents (Figure 5b). This suggests that

191 epigenetic factors may play a role in the response to elevated $\mathrm{CO}_{2}$, and that chromatin

192 and methylation measurements should be included in future studies.

\section{Transgenerational responses to elevated $\mathrm{CO}_{2}$}

195 The within-generation comparisons revealed a large number of DETs in fish that were

196 acutely or developmentally exposed to elevated $\mathrm{CO}_{2}$. By contrast, many of these

197 transcripts exhibited expression levels similar to control levels in fish that were

198 transgenerationally exposed to elevated $\mathrm{CO}_{2}$ (Supplementary Figure 2). A total of 401

199 DETs in the developmental treatment were at control levels in the transgenerational

200 treatment, regardless of parental phenotype (Figure 3b, Supplementary Data 10). The

201 previously mentioned upregulation of histone expression was generally lower in control

202 and transgenerational treatments and higher in the acute and developmental treatments.

203 Furthermore, altered within-generation gene expression patterns, including the $\mathrm{GABA}_{\mathrm{A}}$

204 related genes, were at control levels in the transgenerational treatment. The transcripts 
205 exhibiting recovery patterns, compared with increased expression during developmental 206 exposure, were functionally enriched for microtubule-related pathways (e.g., microtubule

207 proteins; map 1b, map4, futsch, microtubule kinases; mast3, mark3, and microtubule-actin

208 crosslinking factor; macfl, Figure 5c). We also identified an opposite pattern of lower

209 expression levels in the developmental treatment for cytoskeleton related genes (e.g.,

210 tubulin alpha $1 \mathrm{c} ; \mathrm{tublc}$ and microtubule associated protein light chain; map $1 l c 3 b$ ).

212 By comparing within-generation and transgenerational $\mathrm{CO}_{2}$ treatments, we were also able

213 to tease apart a transgenerational-specific transcriptional signature. This refers to

214 transcripts that were at control levels in acute and developmental treatments but were

215 differentially expressed in the transgenerational treatment only. The transgenerational-

216 specific signatures were divergent between offspring from the two parental phenotypes.

217 A larger transgenerational signal was found, represented by 41 transcripts, in offspring of

218 tolerant parents and 8 DETs in offspring of sensitive parents, with none overlapping

219 (Supplementary Data 11). Eleven and one of these transcripts, respectively, showed direct

220 differential expression between the two parental phenotypes in the developmental 221 treatment.

223 Finally, independent of the length of exposure, there were only a few brain transcripts 224 commonly differentially expressed in all elevated $\mathrm{CO}_{2}$ treatments when compared to 225 control fish (Supplementary Figure 3). Only eight and 18 transcripts in offspring of 226 sensitive and tolerant parental phenotypes, respectively, were differentially expressed 227 across all elevated $\mathrm{CO}_{2}$ treatments. When considering long-term treatments (i.e., 
228 excluding acute), 31 and 27 transcripts from offspring of sensitive and tolerant parents,

229 respectively, showed a clear $\mathrm{CO}_{2}$ response (Supplementary Data 12). These $\mathrm{CO}_{2}$-affected

230 transcripts differed in their expression patterns across parental phenotypes, with the 231 exception of $f g f 1, \operatorname{shmt} 2, p c k 1$, arhgef, phdgh and psat that were differentially expressed 232 in various $\mathrm{CO}_{2}$ exposures and common between parental phenotypes (Supplementary 233 Figure $4 \& 5)$.

234 


\section{Discussion}

236 Fundamental changes in the transcriptional landscape of the brain, displayed by

237 numerous differentially expressed genes, were observed in all elevated $\mathrm{CO}_{2}$ treatments.

238 Nevertheless, the specific functional response depended on the duration of exposure. The

239 4-day acute $\mathrm{CO}_{2}$ treatment resulted in the largest treatment-specific response. Several

240 glycoprotein-encoding genes, including neurexophilin (nxphl, 2 and 4) and ependymin

241 (epdl), were overexpressed in acutely-treated fish. These genes play a role in short-term

242 neuronal plasticity, and neurexophilin has recently been linked to GABA receptor subunit

243 expression, revealing an instructive role in configuring GABA receptors ${ }^{28}$. The increased

244 expression of GABA receptor genes in the acutely treated fish could therefore also be

245 driven by an upregulation of $n x p h 1$ and associated inhibitory neural circuits.

247 When fish were reared under elevated $\mathrm{CO}_{2}$ from hatching (i.e., developmental treatment),

248 fewer treatment-specific responses were observed, with most genes downregulated. This

249 was the case for reticulon-4 (rtn4), a neurite growth regulating factor that, in mammals,

250 activates the growth-inhibiting Nogo receptor complex in regenerating axons ${ }^{29}$, thus

251 downregulating growth and inhibiting neuronal plasticity. The function of the Nogo

252 receptor in fish is still unclear, but it was previously associated with embryonic and brain

253 development ${ }^{30}$. Another possible negative effect associated with elevated $\mathrm{CO}_{2}$ during

254 development was the downregulation of the creatine transporter (slc6a8). This could

255 cause a decrease in intracellular creatine, which plays a central role in energy

256 homeostasis ${ }^{31}$. Thus, our results indicate that elevated $\mathrm{CO}_{2}$ exposure early in life could

257 have detrimental effects on development. This is consistent with previous studies 
258 reporting negative effects on growth, development, and survival in juvenile fish exposed 259 to elevated $\mathrm{CO}_{2}{ }^{6,16,32-34}$.

261 Fish exposed to elevated $\mathrm{CO}_{2}$ regulate their intra- and extracellular $\mathrm{pH}$ to avoid acidosis,

262 primarily via $\mathrm{HCO}_{3}{ }^{-}$accumulation ${ }^{16}$. Nilsson and coauthors ${ }^{15}$ suggested this could lead to

263 altered $\mathrm{GABA}_{\mathrm{A}}$ receptor function. Specifically, changes in transmembrane $\mathrm{HCO}_{3}{ }^{-}$and

$264 \mathrm{Cl}^{-}$gradients could lead to a reversal of ion fluxes through the receptor, which could

265 explain the behavioural changes observed in fish upon elevated $\mathrm{CO}_{2}$ exposure ${ }^{35}$. We

266 observed that many GABA-related genes were highly upregulated after acute and

267 developmental exposure to elevated $\mathrm{CO}_{2}$, showing a common within-generation

268 response. This pattern included genes involved in GABA production, all $\mathrm{GABA}_{\mathrm{A}}$

269 receptor subunits, and transporter genes (Figure 4). If $\mathrm{GABA}_{\mathrm{A}}$ receptor function becomes

270 excitatory under elevated $\mathrm{CO}_{2}$, the inhibitory input in neural circuits are lowered, making

271 them overactive. This can trigger futile feedback responses aimed to reduce the over-

272 activity by releasing more GABA and increasing the number of $\mathrm{GABA}_{\mathrm{A}}$ receptors. This

273 will be counter-productive if GABA has started to act excitatory, thus initiating a self-

274 amplifying (vicious) cycle. When CLCN3 and VGAT genes are upregulated, as observed,

275 packing of GABA into synaptic vesicles could increase ${ }^{36,37}$, thereby increasing GABA

276 release. Exacerbation of this vicious cycle also comes from GAT1 (responsible for

277 removing extracellular GABA) being downregulated, which would increase GABA in the

278 synaptic cleft. These changes can explain how a small increase in $\mathrm{CO}_{2}$, causing a

279 moderate change in $\mathrm{Cl}^{-} / \mathrm{HCO}_{3}^{-}$gradients, can be amplified to cause a significant

280 GABAergic dysfunction leading to altered behaviour. We did see one potentially 
281 adaptive GABA related change; upregulation of potassium-chloride co-transporter 2

$282(k c c 2)$ responsible for removing intracellular $\mathrm{Cl}^{-38}$, which could counteract the excitatory

283 action of $\mathrm{GABA}_{\mathrm{A}}$ receptors.

284

285 Epigenetic regulation of gene expression could underpin whole-organism responses to 286 environmental change ${ }^{39}$. Our results suggest regulators influences development under 287 elevated $\mathrm{CO}_{2}$ with an additional effect of parental phenotype. One of the genes that was 288 upregulated in the offspring of sensitive parents compared with tolerant parents, arginine 289 methyltransferase 6 (prmto), is known to methylate CREB Regulated Transcription 290 Coactivator 2 (CRTC2), a transcriptional activator of the gluconeogenic program ${ }^{40,41}$.

291 Upregulated gluconeogesis through the AMPK signaling pathway, which facilitates 292 glucose uptake, would require glucose transporters. Glucose transporters, such as gtrl

293 (gtr10, 3, \& 8), were indeed upregulated in developmentally-treated offspring of sensitive 294 parents. Hence, differential glucose regulation - via selective DNA methylation - could 295 cause differences in the offspring of the two parental groups.

297 Changes to the chromatin landscape and the alternative use of histone variants also 298 influence differences between offspring of tolerant and sensitive parents. Histone variants 299 (e.g., h2az) that were downregulated in the acute $\mathrm{CO}_{2}$ treatment in offspring of tolerant 300 parents and in the developmental treatment in offspring of sensitive parents have been 301 shown to mediate responses to environmental change in animals including fish (e.g., 302 temperature and season $)^{42,43}$. In general, histones and histone modifiers regulate gene 303 expression by controlling chromatin dynamics, making transcription factors more or less 
304 accessible $^{44}$. We found that the general pattern for most histone variants was a decreased 305 expression in the developmental treatment; this pattern has also been identified in a 306 marine invertebrate upon elevated $\mathrm{CO}_{2}$ exposure ${ }^{45}$. Additional evidence for reduced 307 transcriptional repression is the downregulation of several polycomb protein encoding 308 transcripts (e.g., Polycomb Group Ring Finger 2; pcgf2 and SUZ12 Polycomb Repressive 309 Complex 2; suz12b) in the acute and developmental treatments. The polycomb repressive 310 complex chemically modifies histones, for instance, by adding methyl groups, thereby 311 repressing transcription ${ }^{46}$. Thus, downregulation would increase gene expression. Hence, 312 the strong developmental plasticity we see in gene expression is likely controlled in part 313 by DNA methylation and use of histone variants. We also observed that genetic variation 314 and non-genetic (epigenetic) parental effects could, to a certain extent, influence within315 generation control of gene expression of individual fish exposed to elevated $\mathrm{CO}_{2}$.

317 Inheriting an optimized acid-base regulatory system where genes are controlled 318 epigenetically could enhance physiological performance under ocean acidification ${ }^{22,24}$.

319 However, this seems unlikely to occur because transgenerationally $\mathrm{CO}_{2}$-treated fish did 320 not exhibit the aforementioned differential expression of epigenetic-related genes when

321 compared to controls. In fact, it appears that histone genes and many other transcripts 322 specific to within-generation treatments were reversed through transgenerational 323 exposure. Such a recovery pattern was found for multiple microtubule-related genes, 324 implicating cytoskeleton plasticity in response to exposure to near-future $\mathrm{CO}_{2}$ levels, a 325 finding already suggested for invertebrates ${ }^{47,48}$. Cytoskeleton plasticity is directly related 326 to neuronal plasticity ${ }^{49}$, and it seems that within-generation $\mathrm{CO}_{2}$ exposure leads to a 
327 cytoskeletal rearrangement that can aid neuronal plasticity to return to a control state

328 during transgenerational exposure. Further responses to stress via downregulation of

$329 n \operatorname{lrc} 3$ and the hypoxia inducible factor prolyl hydroxylase $2(e g \ln 1)$ and upregulation of

330 the hypoxia inducible factor 2 alpha (epas 1), both important during oxidative stress,

331 could become maladaptive, as we found these expression patterns, even after five months

332 of exposure to elevated $\mathrm{CO}_{2}$. Importantly, such responses seem to also be reversed with

333 transgenerational exposure.

335 The long-term response to elevated $\mathrm{CO}_{2}$, independent of parental phenotype, was linked

336 to glucose metabolism. A role of the brain in regulating glucose homeostasis is becoming

337 evident, but it was only recently shown that increased brain $f g f l$ can promote blood

338 glucose reduction ${ }^{50}$. All previously reported genes involved in transgenerational

339 acclimation to elevated $\mathrm{CO}_{2}{ }^{27}$ were upregulated in our developmental and

340 transgenerational $\mathrm{CO}_{2}$ treatments, suggesting a delayed response to prolonged exposure

341 rather than an immediate adaptive response. Therefore, we propose that the capacity for

342 fish to maintain performance in acidified oceans will depend of their ability to cope with

343 long-lasting $\mathrm{CO}_{2}$ effects. The rebalance of gluconeogenesis and glucose homeostasis,

344 neither of which is compensated for via transgenerational exposure, may be key to

345 adapting to new environmental conditions.

347 Here, by using an integrative genomics approach coupled with a unique experimental 348 design, we tested the response of a coral reef fish to end-of-century $\mathrm{CO}_{2}$ levels and

349 provide further evidence for an important role of altered GABA receptor function in the 
350 response to elevated $\mathrm{CO}_{2}$. In particular we demonstrated a possible vicious feedback 351 cycle exacerbating the GABA pathway reaction to elevated $\mathrm{CO}_{2}$, which can explain the 352 fast neural impairment. Importantly, we identified numerous transcriptional changes in 353 within-generation treatments that returned to baseline levels in fish that were 354 transgenerationally exposed to elevated $\mathrm{CO}_{2}$ levels. This emphasizes the influence of 355 environmental exposure on the parents as well as the parental phenotype in the response 356 of fish to future ocean acidification.

357 


\section{References}

359 1. Doney, S. C., Fabry, V. J., Feely, R. A. \& Kleypas, J. A. Ocean acidification: the 360 other $\mathrm{CO}_{2}$ problem. Ann. Rev. Mar. Sci. 1, 169-92 (2009).

361 2. Kroeker, K. J. et al. Impacts of ocean acidification on marine organisms:

362 quantifying sensitivities and interaction with warming. Glob. Chang. Biol. 19,

$363 \quad 1884-96(2013)$.

364 3. Wittmann, A. C. \& Pörtner, H.-O. Sensitivities of extant animal taxa to ocean 365 acidification. Nat. Clim. Chang. 3, 995-1001 (2013).

366 4. Hamilton, S. L. et al. Species-specific responses of juvenile rockfish to elevated $367 \quad \mathrm{pCO}_{2}$ : From behavior to genomics. PLoS One 12, e0169670 (2017).

368 5. Ries, J. B., Cohen, A. L. \& McCorkle, D. C. Marine calcifiers exhibit mixed 369 responses to $\mathrm{CO}_{2}$-induced ocean acidification. Geology 37, 1131-1134 (2009).

370 6. Stiasny, M. H. et al. Ocean acidification effects on atlantic cod larval survival and 371 recruitment to the fished population. PLoS One 11, e0155448 (2016).

372 7. Clements, J. \& Hunt, H. Marine animal behaviour in a high $\mathrm{CO}_{2}$ ocean. Mar. Ecol. 373 Prog. Ser. 536, 259-279 (2015).

3748 8. Nagelkerken, I. \& Munday, P. L. Animal behaviour shapes the ecological effects 375 of ocean acidification and warming: moving from individual to community-level 376 responses. Glob. Chang. Biol. 22, 974-89 (2016).

377 9. Dixson, D. L., Munday, P. L. \& Jones, G. P. Ocean acidification disrupts the 378 innate ability of fish to detect predator olfactory cues. Ecol. Lett. 13, 68-75 (2010).

379 10. Munday, P. L. et al. Replenishment of fish populations is threatened by ocean 380 acidification. Proc. Natl. Acad. Sci. U. S. A. 107, 12930-4 (2010). 
381 11. Ferrari, M. C. O. et al. Intrageneric variation in antipredator responses of coral reef fishes affected by ocean acidification: implications for climate change projections on marine communities. Glob. Chang. Biol. 17, 2980-2986 (2011).

384 12. Chivers, D. P. et al. Impaired learning of predators and lower prey survival under 385 elevated $\mathrm{CO}_{2}$ : a consequence of neurotransmitter interference. Glob. Chang. Biol. $386 \quad 20,515-522(2014)$.

387 13. Jellison, B. M., Ninokawa, A. T., Hill, T. M., Sanford, E. \& Gaylord, B. Ocean 388 acidification alters the response of intertidal snails to a key sea star predator. Proc.

389 R. Soc. B Biol. Sci. 283, 20160890 (2016).

390 14. Watson, S.-A. et al. Marine mollusc predator-escape behaviour altered by near391 future carbon dioxide levels. Proc. R. Soc. London B Biol. Sci. 281, (2013).

392 15. Nilsson, G. E. et al. Near-future carbon dioxide levels alter fish behaviour by 393 interfering with neurotransmitter function. Nat. Clim. Chang. 2, 201-204 (2012).

394 16. Heuer, R. M. \& Grosell, M. Physiological impacts of elevated carbon dioxide and 395 ocean acidification on fish. Am. J. Physiol. Regul. Integr. Comp. Physiol. 307, $396 \quad$ R1061-84 (2014).

397 17. Vargas, C. A. et al. Species-specific responses to ocean acidification should 398 account for local adaptation and adaptive plasticity. Nat. Ecol. Evol. 1, 84 (2017).

399 18. Sunday, J. M. et al. Evolution in an acidifying ocean. Trends Ecol. Evol. 29, $117-$ $400 \quad 25(2014)$.

401 19. Schlichting, C. D. \& Wund, M. A. Phenotypic plasticity and epigenetic marking: 402 an assessment of evidence for genetic accommodation. Evolution. 68, 656-672 $403 \quad$ (2014). 
404 20. Bonduriansky, R., Crean, A. J. \& Day, T. The implications of nongenetic

405 inheritance for evolution in changing environments. Evol. Appl. 5, 192-201

$406 \quad$ (2012).

407 21. Salinas, S., Brown, S. C., Mangel, M. \& Munch, S. B. Non-genetic inheritance and 408 changing environments. Non-Genetic Inherit. 1, (2013).

409 22. Munday, P. L. Transgenerational acclimation of fishes to climate change and $410 \quad$ ocean acidification. F1000Prime Rep. 6, 99 (2014).

411 23. Miller, G. M., Watson, S.-A., Donelson, J. M., McCormick, M. I. \& Munday, P. L.

412 Parental environment mediates impacts of increased carbon dioxide on a coral reef

413 fish. Nat. Clim. Chang. 2, 858-861 (2012).

414 24. Murray, C., Malvezzi, A., Gobler, C. \& Baumann, H. Offspring sensitivity to 415 ocean acidification changes seasonally in a coastal marine fish. Mar. Ecol. Prog.

$416 \quad$ Ser. 504, 1-11 (2014).

417 25. Welch, M. J. \& Munday, P. L. Heritability of behavioural tolerance to high $\mathrm{CO}_{2}$ in 418 a coral reef fish is masked by non-adaptive phenotypic plasticity. Evol. Appl. 10 $419 \quad$ (7), 682-693 (2017).

420 26. Dupont, S. \& Pörtner, H. Marine science: Get ready for ocean acidification. Nature $421 \quad 498,429-429(2013)$.

422 27. Schunter, C. et al. Molecular signatures of transgenerational response to ocean 423 acidification in a species of reef fish. Nat. Clim. Chang. 6, 1014-1018 (2016).

424 28. Born, G. et al. Modulation of synaptic function through the $\alpha$-neurexin-specific $425 \quad$ ligand neurexophilin-1. Proc. Natl. Acad. Sci. 111, E1274-E1283 (2014).

426 29. Rasmussen, J. P. \& Sagasti, A. Learning to swim, again: Axon regeneration in fish. 

Exp. Neurol. 287, 318-330 (2017).

428 30. Pinzón-Olejua, A., Welte, C., Abdesselem, H., Málaga-Trillo, E. \& Stuermer, C.

429 A. Essential roles of zebrafish rtn4/Nogo paralogues in embryonic development.

$430 \quad$ Neural Dev. 9, 8 (2014).

431 31. Snow, R. J. \& Murphy, R. M. Creatine and the creatine transporter: A review. Mol. $432 \quad$ Cell. Biochem. 224, 169-181 (2001).

433 32. Frommel, A. Y. et al. Severe tissue damage in Atlantic cod larvae under increasing $434 \quad$ ocean acidification. Nat. Clim. Chang. 2, 42-46 (2011).

435 33. Baumann, H., Talmage, S. C. \& Gobler, C. J. Reduced early life growth and 436 survival in a fish in direct response to increased carbon dioxide. Nat. Clim. Chang. $437 \quad 2,38-41(2011)$.

438 34. Frommel, A. Y. et al. Organ damage in Atlantic herring larvae as a result of ocean 439 acidification. Ecol. Appl. 24, 1131-1143 (2014).

440 35. Nilsson, G. E. \& Lefevre, S. Physiological challenges to fishes in a warmer and $441 \quad$ acidified future. Physiology 31, (2016).

442 36. Riazanski, V. et al. Presynaptic CLC-3 determines quantal size of inhibitory 443 transmission in the hippocampus. Nat. Neurosci. 14, 487-494 (2011).

444 37. Ahnert-Hilger, G. \& Jahn, R. CLC-3 spices up GABAergic synaptic vesicles. Nat. $445 \quad$ Neurosci. 14, 405-407 (2011).

446 38. Kaila, K. et al. The K+/Cl- co-transporter KCC2 renders GABA hyperpolarizing 447 during neuronal maturation. Nature 397, 251-255 (1999).

448 39. Turner, B. M. Epigenetic responses to environmental change and their 449 evolutionary implications. Philos. Trans. R. Soc. London B Biol. Sci. 364, (2009). 
450 40. Lerner, R. G., Depatie, C., Rutter, G. A., Screaton, R. A. \& Balthasar, N. A role

451 for the CREB co-activator CRTC2 in the hypothalamic mechanisms linking

452 glucose sensing with gene regulation. EMBO Rep. 10, 1175-81 (2009).

453 41. Han, H.-S. et al. Arginine methylation of CRTC2 Is critical in the transcriptional 454 control of hepatic glucose metabolism. Sci. Signal. 7, (2014).

455 42. Talbert, P. B. \& Henikoff, S. Environmental responses mediated by histone 456 variants. Trends Cell Biol. 24, 642-650 (2014).

457 43. Pinto, R. et al. Seasonal environmental changes regulate the expression of the 458 histone variant macroH2A in an eurythermal fish. FEBS Lett. 579, 5553-5558 $459 \quad(2005)$.

460 44. Feil, R. \& Fraga, M. F. Epigenetics and the environment: emerging patterns and 461 implications. Nat. Rev. Genet. 13, 97 (2012).

462 45. Padilla-Gamiño, J. L., Kelly, M. W., Evans, T. G. \& Hofmann, G. E. Temperature 463 and $\mathrm{CO}_{2}$ additively regulate physiology, morphology and genomic responses of

464 larval sea urchins, Strongylocentrotus purpuratus. Proc. R. Soc. London B Biol. 465 Sci. 280, (2013).

466 46. Di Croce, L. \& Helin, K. Transcriptional regulation by Polycomb group proteins. 467 Nat. Struct. Mol. Biol. 20, 1147-1155 (2013).

468 47. Mukherjee, J. et al. Proteomic response of marine invertebrate larvae to ocean 469 acidification and hypoxia during metamorphosis and calcification. J. Exp. Biol.

$470 \quad$ 216, (2013).

471 48. Kaniewska, P. et al. Major cellular and physiological impacts of ocean 472 acidification on a reef building coral. PLoS One 7, e34659 (2012). 
473 49. Zapara, T. A., Simonova, O. G., Zharkikh, A. A. \& Ratushnyak, A. S. The effects 474 of the dynamic state of the cytoskeleton on neuronal plasticity. Neurosci. Behav. $475 \quad$ Physiol. 30, 347-355 (2000).

476 50. Scarlett, J. M. et al. Central injection of fibroblast growth factor 1 induces 477 sustained remission of diabetic hyperglycemia in rodents. Nat. Med. 22, 800-806 $478 \quad$ (2016).

479 51. Meinshausen, M. et al. The RCP greenhouse gas concentrations and their 480 extensions from 1765 to 2300. Clim. Change 109, 213-241 (2011).

481 52. Collins, M. et al. in Climate Change 2013: The Physical Science Basis. 482 Contribution of Working Group I to the Fifth Assessment Report of the 483 Intergovernmental Panel on Climate Change [Stocker, T.F., D. Qin, G.-K. 484 Plattner, M. Tignor, S.K. Allen, J. Boschung, A. Nauels, Y. Xia, (Cambridge 485 University Press, Cambridge, United Kingdom and New York, NY, USA., 2013). 486 53. Pierrot, D., Lewis, E. \& Wallace, D. MS Excel program developed for $\mathrm{CO}_{2}$ system 487 calculations. ORNL/CDIAC-105a. Carbon Dioxide Inf. (2006).

488 54. Dickson, A. G. \& Millero, F. J. A comparison of the equilibrium constants for the 489 dissociation of carbonic acid in seawater media. Deep Sea Res. Part A. Oceanogr. $490 \quad$ Res. Pap. 34, 1733-1743 (1987).

491 55. Bolger, A. M., Lohse, M. \& Usadel, B. Trimmomatic: a flexible trimmer for 492 Illumina sequence data. Bioinformatics 30, 2114-2120 (2014).

493 56. Andrews, S. FASTQC. A quality control tool for high throughput sequence data. 494 (2010). Available online at:

495 http://www.bioinformatics.babraham.ac.uk/projects/fastqc 
496 57. Kim, D. et al. TopHat2: accurate alignment of transcriptomes in the presence of

497 insertions, deletions and gene fusions. Genome Biol. 14, R36 (2013).

498 58. Li, H. et al. The Sequence Alignment/Map format and SAMtools. Bioinformatics

$499 \quad 25,2078-9(2009)$.

500 59. Anders, S., Pyl, P. T. \& Huber, W. HTSeq - A Python framework to work with

501 high-throughput sequencing data. Bioinformatics 31, 166-169 (2014).

502 60. Love, M. I., Huber, W. \& Anders, S. Moderated estimation of fold change and

503 dispersion for RNA-seq data with DESeq2. Genome Biol. 15, 550 (2014).

504 61. Langfelder, P. \& Horvath, S. WGCNA: an R package for weighted correlation

505 network analysis. BMC Bioinformatics 9, 559 (2008).

506 62. Conesa, A. et al. Blast2GO: a universal tool for annotation, visualization and

507 analysis in functional genomics research. Bioinformatics 21, 3674-6 (2005).

508 63. Wang, Y. E., Kuznetsov, L., Partensky, A., Farid, J. \& Quackenbush, J. WebMeV:

509 A Cloud Platform for Analyzing and Visualizing Cancer Genomic Data. bioRxiv

$510 \quad$ (2017).

511 64. Untergasser, A. et al. Primer3Plus, an enhanced web interface to Primer3. Nucleic

$512 \quad$ Acids Res. 35, W71-4 (2007).

513

514 


\section{Acknowledgments}

516 This study was supported by the Office of Competitive Research Funds OSR-2015-

517 CRG4-2541 from the King Abdullah University of Science and Technology (T.R.,

518 P.L.M., C.S., J.L.R.), the Australian Research Council (ARC) and the ARC Centre of

519 Excellence for Coral Reef Studies (P.L.M, J.L.R.) and the University of Oslo (G.E.N.).

520 We thank the Marine and Aquaculture Research Facilities Unit (JCU), Integrative

521 Systems Biology Laboratory (KAUST), and Biosciences Core Laboratory (KAUST) for

522 support and assistance. Figures 1 to 4 were produced by Xavier Pita, scientific illustrator

523 at King Abdullah University of Science and Technology (KAUST).

524

525 Affiliations

526 KAUST Environmental Epigenetic Program (KEEP), Division of Biological and

527 Environmental Sciences \& Engineering, King Abdullah University of Science and

528 Technology, Thuwal, Kingdom of Saudi Arabia

529 Celia Schunter and Timothy Ravasi

530

531 ARC Centre of Excellence for Coral Reef Studies and College of Marine and

532 Environmental Sciences, James Cook University, Townsville, Queensland, Australia

533 Megan Welch

534

535 ARC Centre of Excellence for Coral Reef Studies, James Cook University, Townsville,

536 Queensland, Australia

537 Jodie L. Rummer, Philip L. Munday 
539 Section for Physiology and Cell Biology, Department of Biosciences, University of

540 Oslo, Oslo NO-0316, Norway

541 Göran E. Nilsson

542

543 Author contributions: M.J.W. and P.L.M designed and managed the fish rearing

544 experiments. M.J.W. performed the adult fish behavioural phenotyping. C.S. prepared the

545 samples for RNA sequencing and analysed transcriptome expression data and performed

546 quantitative real-time PCR expression validation. G.E.N. and J.L.R. assisted in

547 interpreting the expression data. C.S., P.L.M. and T.Ravasi wrote the paper and all

548 authors read, revised, and approved the final manuscript.

549

550 Data availability:

551 All data generated, analysed or used during this study such as the RNA-seq transcriptome

552 sequences and the de novo assembled reference genome have been deposited in NCBI

553 under BioProject ID PRJNA311159

554

555 Competing financial interests

556 The authors declare no competing financial interests. 


\section{Methods}

559 Adult collection and response of adult fish to elevated $\mathrm{CO}_{2}$

560 Adult Acanthochromis polyacanthus (spiny damselfish) were collected as described in

561 Schunter et al. $(2016)^{27}$ in the central Great Barrier Reef, Australia $\left(18^{\circ} 38^{\prime} 24,3^{\prime \prime}\right.$,

$\left.562146^{\circ} 29^{\prime} 31,8^{\prime \prime} \mathrm{E}\right)$ and exposed to $754 \pm 92 \mu$ atm $\mathrm{CO}_{2}$ levels for 7 days before behavioural

563 testing. The behavioural phenotype was determined by exposing the adult fish to

564 conspecific chemical alarm cues (CAC) in a two-chamber flume $(30 \mathrm{~cm} \times 13 \mathrm{~cm})$, where

565 time spent in the CAC was recorded. A 1:1 ratio of adult CAC donor fish to adult test fish

566 was used. Donor fish were held in control conditions until it was euthanized by a quick

567 blow to the head. To generate CAC, superficial cuts to both sides of the body were made

568 after euthanization of the donor fish. The fish was then rinsed with $60 \mathrm{ml}$ of control

569 water ${ }^{27}$, and the rinse water was added to $10 \mathrm{~L}$ of elevated $\mathrm{CO}_{2}$ seawater. Elevated $\mathrm{CO}_{2}$

570 water including $\mathrm{CAC}$ and elevated $\mathrm{CO}_{2}$ control water were fed into the flume at a

571 constant rate of $450 \mathrm{ml}$ per minute. Each behavioural trial was run for 9 minutes (2

572 minutes habituation, 2 minutes recording, 1 minute switch for water sides, where the fish

573 was recentered at the end of this minute. The 2 minutes habituation and 2 minutes

574 recording was then repeated), and the location of the fish was recorded every 5 seconds.

575 Adult fish exhibited a large variation in behavioural responses when tested for chemical

576 alarm cue (CAC) recognition. These responses ranged from a normal aversion behaviour

577 with little time spent in the CAC to the opposing behavior, where fish spent most of their

578 time in CAC. We considered those fish exhibiting an aversion to $\mathrm{CAC}$ to be behaviorally

579 'tolerant' ( $<$ than $30 \%$ of the trial in CAC) and those exhibiting an attraction to CAC 580 under elevated $\mathrm{CO}_{2}$ to be behaviorally 'sensitive' ( $>$ than $70 \%$ of the trial in $\mathrm{CAC}$ ). About 
$58138 \%$ of the randomly collected fish within the population could be assigned to the

582 tolerant or sensitive groups (Supplementary Data 13). Behavioural sensitivity and fish

583 size were then used to form breeding pairs with individuals of the same sensitivity (i.e.,

584 tolerant male with tolerant female). This project was completed under James Cook

585 University (JCU) ethics permit A1828.

586

587 Experimental design

588 Breeding pairs were held in $40 \mathrm{~L}$ aquaria, with 3 tolerant and 3 sensitive pairs in control 589 conditions $(414 \pm 46 \mu \mathrm{atm})$ and 2 tolerant and 3 sensitive pairs in elevated $\mathrm{CO}_{2}$ conditions 590 (754 $\pm 92 \mu \mathrm{atm}$, Supplementary Data 13). Breeding pairs were acclimated to their 591 respective conditions for three months prior to the breeding season. Offspring clutches 592 from breeding pairs were immediately removed from parental tanks after hatching and 593 placed into control or elevated $\mathrm{CO}_{2}$ conditions. A total of four combinations between 594 parental and offspring conditions were processed with several parental pairs for each 595 combination to avoid a family effect (Figure 1, Supplementary Data 13). Offspring 596 conditions were: a) control conditions, b) acute elevated $\mathrm{CO}_{2}$ treatment, in which 597 offspring developed in control conditions but were acutely exposed to elevated $\mathrm{CO}_{2}$ for 598 the last 4 days before sacrificing, c) developmental elevated $\mathrm{CO}_{2}$ treatment, in which 599 offspring were immediately placed into elevated $\mathrm{CO}_{2}$ after hatching and d) 600 transgenerational elevated $\mathrm{CO}_{2}$ treatment where parents and offspring were exposed to 601 elevated $\mathrm{CO}_{2}$. Offspring were kept in their respective conditions (Figure 1) and sacrificed 602 at the age of 5 months. 


\section{$604 \mathrm{CO}_{2}$ treatment}

605 Experimental procedures followed those described by Welch and Munday $(2017)^{25}$.

606 Briefly, two 10,000 L recirculating aquarium systems were each set to a different $\mathrm{pH}$ and

607 corresponding $\mathrm{CO}_{2}$ level: a current-day control (414 $\left.\pm 46 \mu \mathrm{atm}\right)$ and an end of century

608 elevated $\mathrm{CO}_{2}$ treatment $(754 \pm 92 \mu \mathrm{atm})^{51,52}$. An Aqua Medic AT Control System (Aqua

609 Medic, Germany) was used to dose $\mathrm{CO}_{2}$ into a 3,000 L sump to maintain the desired $\mathrm{pH}$

610 in the elevated $\mathrm{CO}_{2}$ treatment. An identical sump on the control system was not dosed

611 with $\mathrm{CO}_{2}$. Control and elevated $\mathrm{CO}_{2}$ water were then delivered to the holding aquaria at

$6121.5 \mathrm{~L}$ per minute. Temperature and $\mathrm{pH}_{\mathrm{NBS}}$ were measured daily in randomised tanks.

613 Salinity and total alkalinity were measured weekly. Total alkalinity was measured by

614 Gran Titration (Metrohm 888 Titrando Titrator Metrohm AG, Switzerland) using

615 certified reference material from Dr. A.G. Dickson (Scripps Institution of

616 Oceanography). $\mathrm{pCO}_{2}$ was then calculated in $\mathrm{CO}_{2} \mathrm{SYS}^{53}$, using constants from Dickson 617 and Millero $(1987)^{54}$.

618

$619 R N A$ and transcriptome expression analyses

620 Fish brains were immediately dissected out after euthanization, snap frozen with liquid 621 nitrogen, and stored at $-80^{\circ} \mathrm{C}$. Whole frozen fish brains were then homogenized in RT622 Plus Buffer for 30 second in a Fisher bead beater with single-use silicon beads, and total 623 RNA was extracted with AllPrep DNA/RNA Mini Kits (Quiagen). The RNA quality was 624 evaluated on the nanodrop and the Agilent Tape reader, and only minimum RNA 625 integrity values (RIN) of 8 were accepted. Extracted RNA was converted into cDNA and 626 prepped for Illumina sequencing with a TruSeq RNA Illumina Library Prep Kit. Libraries 
627 were then sequenced on an Illumina HiSeq 2500 paired end to the length of $100 \mathrm{bp}$ at

628 Macrogen, South Korea. Raw reads were inspected and quality trimmed to a minimum

629 Phred score of 30 with FastQC and Trimmomatic respectively ${ }^{55,56}$. High quality reads

630 were mapped against the de novo assembled genome reference using Tophat $2^{57}$ with

631 bowtie2 very-sensitive mode and providing the coordinates of the reference based

632 annotated transcriptome. The A. polyacanthus de novo genome assembly and annotation

633 have been previously described ${ }^{27}$. The bam files resulting from the mapping step were

634 then sorted with samtools ${ }^{58}$ and read counts were extracted by using an HT-seq script ${ }^{59}$

635 adding exon read counts to receive transcript-based read count values. Differential 636 expression was statistically evaluated with DEseq $2^{60}$ in Bioconductor version 3.2 in $\mathrm{R}$

637 3.2.1 through pair-wise treatment comparisons. Comparisons between the different 638 treatments were performed by comparing the expression of acute, developmental, and 639 transgenerational samples for each parental phenotype separately against the control 640 samples. Differential expression was evaluated between the different treatments, but the

641 expression levels of the two parental phenotypes were also directly compared for each

$642 \mathrm{CO}_{2}$ treatment. The significance level for differential expression was set to an FDR

643 adjusted p-value of $<0.05$ with additional filters of a minimum $\log 2$ fold expression of

6440.3 and standard deviation correction $(\mathrm{SD}<\mathrm{Mean})$. Gene expression patterns across

645 different treatments were based on significant differential expression in all pairwise 646 comparisons.

648 To evaluate a potential family effect within the parental phenotypes, we compared 649 treatments in which full siblings were exposed (comparison of control and acute as well 
650 as developmental treatments for offspring of tolerant and sensitive parents). We used a

651 model comparison approach. First, differential expression was measured accounting for

652 treatment effect only, then family line was added as a factor and differential expression

653 compared. Finally, the full (treatment+family) model was compared directly with the

654 reduced model (treatment only) (Supplementary Data S14).

655

656 After stringent filtering of significant differential expression assignment, we further 657 accounted for false positive assignment through randomization. This was done on the 658 acutely and developmentally treated samples comparing the two different parental

659 phenotypes. For each $\mathrm{CO}_{2}$ treatment parental phenotype was randomly assigned to a gene

660 expression profile and gene expression analysis was rerun. This was repeated 10 times for

661 the acute and the developmental treatments (Supplementary Data S15).

662

663 To improve insight into the complex dataset, we performed a weighted gene-correlation

664 network analysis with the WGCNA package (version 1.6) in $\mathrm{R}^{61}$. We used the DEseq2

665 normalized dataset of raw counts of all 72 samples included in the study. Gene

666 expression data was then variance stabilized, and transcripts with low read counts were

667 removed. Soft-thresholding power was evaluated and the highest value was accepted for

668 network construction (pow=9). This approach was used to approximate a scale free

669 topological network (TOM), which was constructed following these parameters:

670 TOMtype $=$ "assigned', minModuleSize $=30$, mergeCutHeight $=0.25$. TOM was then used

671 to create a cluster dendogram. Transcripts clustered within one colour module were then 
672 extracted if the module had more than 500 transcripts and compared with the

673 differentially expressed gene analysis (Supplementary Figure $6 \&$ 7).

674

675 Blast annotations of the reference-based transcriptome and an Interpro scan were 676 imported into Blast2 $\mathrm{GO}^{62}$ to retrieve Gene Ontology terms and KEGG pathways.

677 Functional enrichment analyses were performed for differentially expressed genes as well

678 as global network clusters with Fisher's exact tests (FDR $<0.05)$. All tests were

679 performed on the different differential gene expression models, and results presented

680 were significantly enriched functions found with both models. Graphical representations

681 (i.e., heat maps, bubble graphs, and bar plots) were produced in R 3.3.1. A Principle

682 Component Analysis (PCA) was performed with the cloud platform $\mathrm{WebMeV}^{63}$ using the 683 normalized expression of acutely and developmentally treated samples.

684

685 qRT-PCR validation of $R N A-$ seq results

686 Quantitative Realtime PCR was performed on two sets of samples to evaluate all the 687 different experimental treatment groups. We compare control samples with 688 transgenerational elevated $\mathrm{CO}_{2}$ exposed fish from behaviourally tolerant as well as 689 sensitive parents. We also examined the qPCR gene expression for acutely and 690 developmentally elevated $\mathrm{CO}_{2}$ treated fish for both parental pairs and compare the 691 relative expression between treatments with the RNAseq expression differences

692 (Supplementary Figure 8). For each treatment group, two biological samples were 693 selected, which were from the same treatment, but additional biological individuals than 694 those sequenced via RNAseq. Primers were designed using the genome sequence of the 
695 respective transcript of interest with Primer3Plus ${ }^{64}$, which was checked in NCBI Primer-

696 BLAST for specificity and HPSF purified by Sigma (Sigma-Aldrich, Germany). Using

697 the high capacity reverse transcription kit by ABI (Applied Biosystems) 550ng of RNA

698 for each sample were reverse transcribed and 15ng of cDNA was used for each reaction

699 with three replicate reactions with specified reaction details ${ }^{27}$. For analysis, the livak

700 method was used and Delta Delta CTs were calculated by normalizing the CTs against

701 three housekeeping genes. Eight comparisons were performed: Offspring of tolerant and

702 sensitive parents were compared at the 1) control $\mathrm{CO}_{2}$ levels, 2) acute high $\mathrm{CO}_{2}$ levels, 3)

703 developmentally high $\mathrm{CO}_{2}$ levels and 4) transgenerational high $\mathrm{CO}_{2}$ levels. Treatments

704 effect were compared between acute and developmental treatments for 5) offspring of

705 tolerant parents and 6) offspring of sensitive parents. Control levels and

706 transgenereational treatment were compared for 7) offspring of tolerant parents and 8)

707 offspring of sensitive parents. Six out of eight genes used for validation were highly

708 correlated and hence showed the same expression pattern in qRT-PCR as found with

709 RNAseq (Pearson's product-moment correlation, $\mathrm{p}<0.001$ ). Transcript expression of $n f i l 3$

710 showed an almost significant correlation (Pearson's product-moment correlation, $\mathrm{p}<0.08$ ),

711 whereas shmt1 did not correlate (Pearson's product-moment correlation, $\mathrm{p}=0.5$ ).

712 However, correlation improves when removing one comparison (HC_S, Pearson's

713 product-moment correlation, $\mathrm{p}=0.1$ ). This high percentage of validation shows that the

714 RNAseq results can be replicated not only with a different method, but also with different

715 biological samples from the same treatment and therefore the observed RNAseq

716 expression pattern is clearly linked to the treatment. 
720 Figure 1. Experimental design. Elevated $\mathrm{CO}_{2}$ (green) was set at 750 uatm, simulating end

721 of century $\mathrm{CO}_{2}$ projections. Behaviourally tolerant and sensitive parents were phenotyped

722 based on their response to chemical alarm cues (CAC) after exposure to elevated $\mathrm{CO}_{2}$ : tolerant

723 adults exhibited a normal response to $\mathrm{CAC}$ in an elevated $\mathrm{CO}_{2}$ environment whereas sensitive

724 parents exhibited an impaired response to CAC. Offspring of parental pairs were then reared

725 in three different $\mathrm{CO}_{2}$ treatments until the age of 5 months These three treatments were: current

726 day $\mathrm{CO}_{2}$ levels as the control (control), fish reared under control conditions with 4 days

727 exposure to elevated $\mathrm{CO}_{2}$ at 5 months of age (acute treatment), and fish reared under elevated

$728 \mathrm{CO}_{2}$ from hatching until 5 months of age (developmental treatment). Control, acute, and

729 developmentally treated fish were siblings from three different parental pairs for both tolerant

730 and sensitive parental phenotypes. The final treatment (transgenerational treatment) consisted

731 of offspring reared in elevated $\mathrm{CO}_{2}$ from hatching until 5 months of age that were from parents

732 maintained in elevated $\mathrm{CO}_{2}$ for breeding.

734 Figure 2. Global differential gene expression patterns between treatments. Numbers

735 of significantly differentially expressed transcripts between pairwise comparisons of $\mathrm{CO}_{2}$

736 treatments as well as between different parental behavioural phenotypes $(\mathrm{T}=$ tolerant

737 parents, $\mathrm{S}=$ sensitive parents). The overlap between blue and green ( $\mathrm{T}$ and $\mathrm{S}$ ) represent the

738 transcripts that are directly differentially expressed between the offspring of different

739 parental phenotypes.

740 
741 Figure 3. Functional enrichment analysis of differentially expressed genes across

$742 \mathrm{CO}_{2}$ rearing treatments that were significant in both differential gene expression

743 models $(\mathrm{C}=$ control, $\mathrm{A}=$ acute, $\mathrm{DEV}=$ developmental, $\mathrm{TRANS}=$ transgenerational $)$ and

744 different behavioural parental phenotypes, $(\mathrm{T}=$ tolerant, $\mathrm{S}=$ sensitive $) . \mathrm{A})$

745 Overrepresented gene ontologies and B) underrepresented gene ontologies (significantly

746 more or less of this GO category in comparison to the compared treatment). The colour of

747 the circles represents the enrichment significance, and size of circles is proportional to the

748 number of enriched genes.

749

750 Figure 4. Gamma-aminobutyric acid (GABA) signaling pathway in the synapse

751 between a pre- and postsynaptic neuron. Many pathway components showed

752 differential expression in response to $\mathrm{CO}_{2}$ treatments. The insert highlights the proposed

753 increase of GABA release due to increased GABA packing in synaptic vesicles ${ }^{37}$.

754 (Adapted from KEGG pathways). GAD= Glutamate decarboxylase 1, VGAT= GABA

755 and glycine transporter, $\mathrm{CLCN} 3=$ Chloride voltage-gated channel $3, \mathrm{KCC} 2=$ Neuronal $\mathrm{K}$ -

$756 \mathrm{Cl}$ cotransporter, GAT1 $=$ GABA transporter $1, \mathrm{CACNA} 1 \mathrm{~A}=$ Brain calcium channel 1 ,

$757 \quad \mathrm{GABAAR}=\mathrm{GABA}_{\mathrm{A}}$ receptor subunits alpha, beta $\&$ gamma.

758

759 Figure 5. Expression pattern of histone-related transcripts across all $\mathbf{C O}_{2}$

760 treatments. Expression levels of a) core histones, b) differential expression of histone-

761 related transcripts between developmentally $\mathrm{CO}_{2}$ treated fish from tolerant and sensitive

762 offspring and c) microtubule-related transcripts. $\mathrm{S}=$ sensitive, $\mathrm{T}=$ tolerant, $\mathrm{C}=$ control,

$763 \mathrm{~A}=$ acute, $\mathrm{DEV}=$ developmental, TRANS=transgenerational. 